\title{
RECENT DEVELOPMENTS OF THE BLOCH-MODIFIED LADDER THEORY
}

\author{
H. SORMANN \\ Institut für Kernphysik, Technische Universität Graz \\ Petersgasse 16, $8010 \mathrm{Graz}$, Austria
}

\begin{abstract}
One of the possible theoretical approaches for desribing the physics of electron-positron pairs in the inhomogeneous electron gas is the so-called "quasi-free" Bloch-modified ladder theory. Despite the success of this approach, it contains two very serious deficiencies, namely the complete neglect of the Bloch character of the electron and positron scattering states and of the electron-positron interaction potential. In this contribution, the importance of these Bloch effects for the Bloch-modified ladder theory results, especially for the momentum dependence of the positron enhancement in $d$-band metals, is demonstrated for the first time.

PACS numbers: 78.70.Bj, 71.25.Pi
\end{abstract}

\section{Introduction}

For theoretical investigations on enhancement effects in the inhomogeneous electron gas, there exist two quite different concepts: (i) The Bloch-modified ladder (BML) theory: this approach and its different approximations like our "quasi-free" BML (QF-BML) are described in several papers [1-5] and are shortly reviewed in Sec. 2 of this contribution. (ii) A second very effective approach to include electron-positron correlation into the independent particle model (IPM) is based on the use of local enhancement factors $\gamma$ in the IPM rate formula, and it is therefore called the local density approximation (LDA) [6-8].

In principle, the BML and the LDA approaches lead to comparable results, but they also show significant differences. What concerns the momentum dependence of the $d$-state enhancement, both theories show a negative slope of the corresponding curves, but this effect is significantly more pronounced for the BML than for the LDA approach. There are some indications that this behaviour of the QF-BML approach comes from its complete neglect of the local aspects of the electron-positron interaction, and it is one of the aims of the work presented here to prove or to disprove this opinion.

Sections 2 and 3 of this paper give a concise summary on the theory of the BML approach and the matrix of the effective interaction potential for an inhomogeneous electron gas. Some details on the numerical work are summarized in Sec. 4. The final sections 5 and 6 contain a discussion about the results of our investigation and a short conclusion. 


\section{Theory}

The starting point of this investigation is the well-known formula for the momentum dependent two-particle annihilation rate [1]:

$$
R(p)=\frac{\lambda}{\Omega}(-\mathrm{i})^{2} \int_{\Omega} \mathrm{d}^{3} x \mathrm{~d}^{3} y \exp [-\mathrm{i} p \cdot(x-y)] G_{\mathrm{ep}}\left(x t, x t ; y t^{+}, y t^{+}\right),
$$

where $\lambda / \Omega$ means the Sommerfeld rate, $\hbar p$ is the photon-pair momentum, and $G_{\text {ep }}$ represents the zero-temperature electron-positron Green's function. Following Kahana, $G_{\text {ep }}$ can be expanded in a series of ladder diagrams $(x \equiv x t$ etc.)

$$
\begin{aligned}
& G_{\mathrm{ep}}\left(x, x^{\prime} ; y, y^{\prime}\right)=G_{\mathrm{e}}(x, y) G_{\mathrm{p}}\left(x^{\prime}, y^{\prime}\right) \\
& \quad+\int \mathrm{d} \xi \mathrm{d} \eta G_{\mathrm{e}}(x, \xi) G_{\mathrm{p}}\left(x^{\prime}, \eta\right) v_{\mathrm{ep}}(\xi, \eta) G_{\mathrm{ep}}\left(\xi, \eta ; y, y^{\prime}\right)
\end{aligned}
$$

with $G_{\mathrm{e}}$ and $G_{\mathrm{p}}$ as the single-particle Green's functions of the electron and positron, respectively, and $v_{\text {ep }}$ as the effective interaction potential between these particles. For a realistic theory of the annihilation process in real metals, it is necessary to describe both the electron and positron propagator and the interaction potential on the basis of Bloch functions. By doing so, one gets the so-called Bloch-modified ladder theory, as it has been at first performed by Carbotte [1]. If one expands the electron and positron Bloch wave functions with respect to plane waves, one gets

$$
\begin{aligned}
& R(p+G)=2 \frac{\lambda}{\Omega} \sum_{n} \Theta\left(\varepsilon_{\mathrm{F}}-\varepsilon_{n} p\right)\left\{\sum_{\boldsymbol{K}} a_{n} p(\boldsymbol{K}) b_{0}(\boldsymbol{G}-\boldsymbol{K})+\right. \\
& \quad+\frac{1}{\Omega^{2}} \sum_{i} \sum_{k} \Theta\left(\varepsilon_{i k}-\varepsilon_{\mathrm{F}}\right) \sum_{j}\left(\varepsilon_{n} p-\varepsilon_{i k}+\varepsilon_{0}^{+}-\varepsilon_{j q}^{+}\right)^{-1} \chi_{i j}^{\mathrm{BM}}(k) \\
& \quad \times \sum_{\boldsymbol{G}_{1}} \sum_{\boldsymbol{G}_{2}} v_{\boldsymbol{G}_{1}, G_{2}}^{\mathrm{ep}}(\boldsymbol{g})\left[\sum_{\boldsymbol{K}_{2}} a_{n} p\left(K_{2}\right) a_{i k}\left(K_{2}-G_{1}+L\right)\right] \\
& \left.\quad \times\left[\sum_{\boldsymbol{K}_{3}} b_{0}\left(\boldsymbol{K}_{3}\right) b_{j} \boldsymbol{q}\left(\boldsymbol{K}_{3}+\boldsymbol{G}_{2}\right)\right]\right\}^{2} .
\end{aligned}
$$

An important quantity in this equation is the Bloch-modified Bethe-Goldslone amplitude $\chi^{\mathrm{BM}}$ which is the solution of a relatively complicated integral equation. For this paper, it is sufficient to know the first term of $\chi$, namely

$$
\chi_{i, j}^{\mathrm{BM}}(k)=\sum_{K_{1}} a_{i k}\left(K_{1}\right) b_{j} q\left(G-K_{1}+L\right)+\text { higher terms }
$$

In Eqs. (1) and (2), the photon-pair momentum is defined as a sum of the vector $\boldsymbol{p}$ (element of the first Brillouin zone BZ) and a reciprocal- lattice vector $\boldsymbol{G}$. The $a_{n} p\left(\varepsilon_{n} p\right)$ and $b_{0}\left(\varepsilon_{0}^{+}\right)$mean the Fourier coefficients (energies) of the occupied electron and positron Bloch states; the non-occupied electron (positron) states are given by the Fourier coefficients and eigenenergies $a_{i k}\left(b_{j} q\right)$ and $\varepsilon_{i k}\left(\varepsilon_{j} q\right)(i, j$ are band indices, $k, q$ - vectors of the first BZ). The vector $q$ and the reciprocal-lattice vector $L$ are defined by $q=p-k-L \in \mathrm{BZ} . v_{G_{1}, G_{2}}^{\mathrm{ep}}(q)$ in Eq. (1) represents the static approximation of the matrix of the effective electron-positron potential (see Sec. 3). 
In order to obtain a more feasible formulation of the original BML theory, Sormann and Puff [2] published the "quasi-free" BML approach w\}.ich includes the two following simplifications:

(i) Only the occupied electron and positron states used in Eqs. (1) and (2) are considered as Bloch waves, whereas all non-occupied $\mathrm{e}^{-}$and $\mathrm{e}^{+}$scattering states are described as plane waves (like in the original Kahana theory).

(ii) The Bloch character of the electron-positron interaction quantity $v^{\mathrm{ep}}$ is completely neglected by using the approximation

$$
v_{G_{1}, G_{2}}^{\mathrm{ep}}(q) \approx-\delta_{G_{1}, G_{2}} v_{C}\left(q+G_{1}\right)\left[1-v_{C}\left(q+G_{1}\right) P\left(q+G_{1}\right)\right]^{-1}
$$

with $v_{\mathrm{C}}$ as the bare Coulomb potential and $P(q)=P(|q|)$ as the (static) polarization function of the homogeneous electron theory.

Despite some successful applications of the QF-BML approach $[2,10]$, the simplifications of the theory may cause serious problems, and it is therefore highly desirable to investigate how the BML results are influenced by these approximations. In order to reduce the considerable computer time for these calculations, we decided to start our investigation with the first two terms of the ladder expansion of $G_{\text {ep }}$ which means that only the first term of the Bethe-Goldstone integral equation (2) is inserted into the rate formula (1). Under these circumstances, one obtains

$$
R(p+G)=2 \frac{\lambda}{\Omega} \sum_{n} \theta\left(\varepsilon_{F}-\varepsilon_{n} p\right)\left\{\left[t_{n}^{(0)}(p ; G)\right]^{2}+2 t_{n}^{(0)}(p ; G) t_{n}^{(1)}(p ; G)\right\}
$$

with

$$
\begin{aligned}
& t_{n}^{(0)}(p ; G)=\sum_{K} a_{n} p(K) b_{0}(G-K) \\
& t_{n}^{(1)}(p ; G)=-\frac{1}{\Omega^{2}} \sum_{i} \sum_{k} \theta\left(\varepsilon_{i k}-\varepsilon_{F}\right) \sum_{j}\left[\sum_{K_{1}} a_{i k}\left(K_{1}\right) b_{j} q\left(G-K_{1}+L\right)\right] \\
& \times\left(\varepsilon_{i k}-\varepsilon_{n} p+\varepsilon_{j q}^{+}-\varepsilon_{0}^{+}\right)^{-1} \\
& \times \sum_{G_{1}} \sum_{G_{2}} v_{G_{1}, G_{2}}^{\mathrm{ep}}(q)\left[\sum_{K_{2}} a_{n} p\left(K_{2}\right) a_{i k}\left(K_{2}-G_{1}+L\right)\right] \\
& \times\left[\sum_{K_{3}} b_{0}\left(K_{3}\right) b_{j} q\left(K_{3}+G_{2}\right)\right] .
\end{aligned}
$$




\section{The matrix of the effective potential}

Due to the importance of this quantity for the investigations presented here, we give a very brief summary of this topic. For more details, see for example Ref. [11].

In an inhomogeneous electron sea, the effective (screened) electron-positron interaction potential can be defined by its Fourier transform

$$
\begin{gathered}
v^{\mathrm{eP}}\left(\boldsymbol{x}, \boldsymbol{y} ; \boldsymbol{t}_{\boldsymbol{x}}-\boldsymbol{t}_{y}\right)=\frac{1}{2 \pi \Omega^{2}} \sum_{k} \exp [\mathrm{i} k \cdot(\boldsymbol{x}-\boldsymbol{y})] \sum_{\boldsymbol{G}_{1}} \sum_{\boldsymbol{G}_{2}} \exp \left(\mathrm{i} \boldsymbol{G}_{1} \cdot \boldsymbol{x}\right) \\
\quad \times \exp \left(-\mathrm{i} \boldsymbol{G}_{2} \cdot \boldsymbol{y}\right) \int_{-\infty}^{+\infty} \mathrm{d} \omega \exp \left[-\mathrm{i} \omega\left(t_{x}-t_{y}\right)\right] v_{\boldsymbol{G}_{1}, \boldsymbol{G}_{2}}^{\mathrm{ep}}(\boldsymbol{k} ; \omega),
\end{gathered}
$$

where $k$ is a vector of the first $\mathrm{BZ}$, and $G_{1}, G_{2}$ are vectors of the reciprocal lattice. Equation (7) shows immediately that all local aspects of the potential are given by the non-diagonal elements (NDE) of ${ }^{v} \boldsymbol{G}_{1}, \boldsymbol{G}_{2}$. In analogy to the homogeneous electron gas, the matrix of the effective potential in the $(k, \omega)$ space can be written as

$$
v_{\boldsymbol{G}_{1}, \boldsymbol{G}_{2}}^{\mathrm{ep}}(k, \omega)=-\Omega \frac{4 \pi e^{2}}{\left|k+G_{1}\right|\left|k+G_{2}\right|}[\kappa(k, \omega)]_{\boldsymbol{G}_{1}, \boldsymbol{G}_{2}}^{-1}
$$

where $\kappa^{-1}$ means the inverse of the dielectric matrix

$$
\kappa_{G_{1}, G_{2}}(k, \omega)=\delta_{G_{1}, G_{2}}-\frac{4 \pi e^{2}}{\left|k+G_{1}\right|\left|k+G_{2}\right|} P_{G_{1}, G_{2}}(k, \omega) .
$$

In this equation, $P$ represents the polarization matrix of the system. The simplest approximation of the matrix $P$ is the random phase approximation (RPA), and the static form of this quantity (we neglect all dynamical effects of the polarization, as it is frequently done in positron physics) reads

$$
\begin{aligned}
& P_{G_{1}, G_{2}}^{\mathrm{RPA}}(k, 0)=P_{G_{1}, G_{2}}^{\mathrm{RPA}}(k)=-\frac{4}{\Omega} \wp \sum_{n} \sum_{k^{\prime}} \Theta\left(\varepsilon_{\mathrm{F}}-\varepsilon_{n k^{\prime}}\right) \sum_{m} \Theta\left(\varepsilon_{m} \boldsymbol{q}-\varepsilon_{\mathrm{F}}\right) \\
& \times\left(\varepsilon_{m} \boldsymbol{q}-\varepsilon_{n k^{\prime}}\right)^{-1} M_{n k^{\prime} ; m \boldsymbol{q}}\left(k, G_{1}\right) M_{n k^{\prime} ; m \boldsymbol{q}}^{*}\left(k, G_{2}\right)
\end{aligned}
$$

with

$$
M_{n k^{\prime} ; m q}(k, G)=\sum_{K} a_{n k^{\prime}}(K) a_{m} \boldsymbol{q}(K-G+L),
$$

where the vectors $q$ and $L$ are defined by $q \equiv k^{\prime}-k-L \in \mathrm{BZ}$.

The polarization of the electron gas will be especially determined by the density of electron states (DOS) nearby the Fermi surface. With other words: local effects of the polarization will be strong if the DOS around $\varepsilon_{F}$ is dominated by localized electron states like $d$ states. For the metals investigated for this paper $(\mathrm{Na}, \mathrm{Cu}$, and $\mathrm{V})$, the situation is as follows: for sodium, the DOS nearby $\varepsilon_{\mathrm{F}}$ is characterized by "nearly-free" $s$ states. This is also (at least approximately) the case for copper whose $d$ bands lie considerably below $\varepsilon_{\mathrm{F}}$. Therefore, for $\mathrm{Na}$ and $\mathrm{Cu}$, one would expect a rather homogeneous polarization behavior with only small $\mathrm{NDE}$ within the polarization matrix. For vanadium, however, the DOS around $\varepsilon_{\mathrm{F}}$ 
is dominantly due to $d$ states, and the local effects of the polarization will be large. This is illustrated in Figs. 1-3 where we show the momentum dependence of the $P_{0,0}$ element along the $(100)$ direction together with the corresponding homogeneous polarization functions.

\section{Numerical details}

The evaluation of Eqs. (6) and (10) requires numerical integrations with respect to $k\left(k^{\prime}\right)$ within the first BZ. For this task, we used the well-known integration method by Gilat and Raubenheimer [12] in connection with the $\boldsymbol{k} \cdot \boldsymbol{p}$ interpolation method and a Monte Carlo integration program. For our calculations on bcc $\mathrm{Na}$ and $\mathrm{V}$ and $\mathrm{fcc} \mathrm{Cu}$, we took up to 240 primary $k$ points within the irreducible wedge (IW) of the corresponding BZ where the electron and positron eigenenergies and wave functions were determined by an augmented plane wave (APW) scheme. To save computer time, in all our calculations, the symmetry properties of the crystal lattices were carefully taken into account.

\section{Annihilation rates - results and discussion}

Some of the results of our investigation are summarized in Figs. 4-7. In contrast to the title of this section, these figures do not show the annihilation rates themselves but the corresponding enhancement curves which are defined by

$$
E_{n}(p+G)=\Theta\left(\varepsilon_{\mathrm{F}}-\varepsilon_{n} p\right)\left[1+2 \frac{t_{n}^{(1)}(p ; G)}{t_{n}^{(0)}(p ; G)}\right]
$$

with $G=(000)$, i.e. umklapp processes are not taken into account. In order to make the following discussions easier, we start with some general remarks about Figs. 4-7.

All curves indicated with (1) belong to results of the QF-BML. All curves indicated with (2) belong to BML results including Bloch scattering states, but with a complete neglect of the Bloch characteristics of the polarization (compare Eq. (3)): these enhancement curves are called "homogeneous curves". All curves indicated with (3) belong to BML results including Bloch scattering states and an interaction potential which contains only the diagonal elements (DE) of the potential matrix from Eq. (8): these curves are called "DE curves". All curves indicated with (4) belong to BML results with Bloch scattering states and the complete interaction potential matrix from Eq. (8).

\subsection{Bloch characteristics in scattering states}

In a previous paper about this topic [9], we spoke out that metals like vanadium show a large difference between the enhancement curves belonging to a PW or an APW approximation of the excited states. This statement is supported by our new results on copper. As it is shown in Figs. 6 and 7, the QF-BML enhancement curves (1) and the corresponding homogeneous curves (2) are significantly different (compare also Figs. 2 and 3 in [9]). What concerns the momentum dependence of the enhancement curves with and without Bloch scattering states, we 

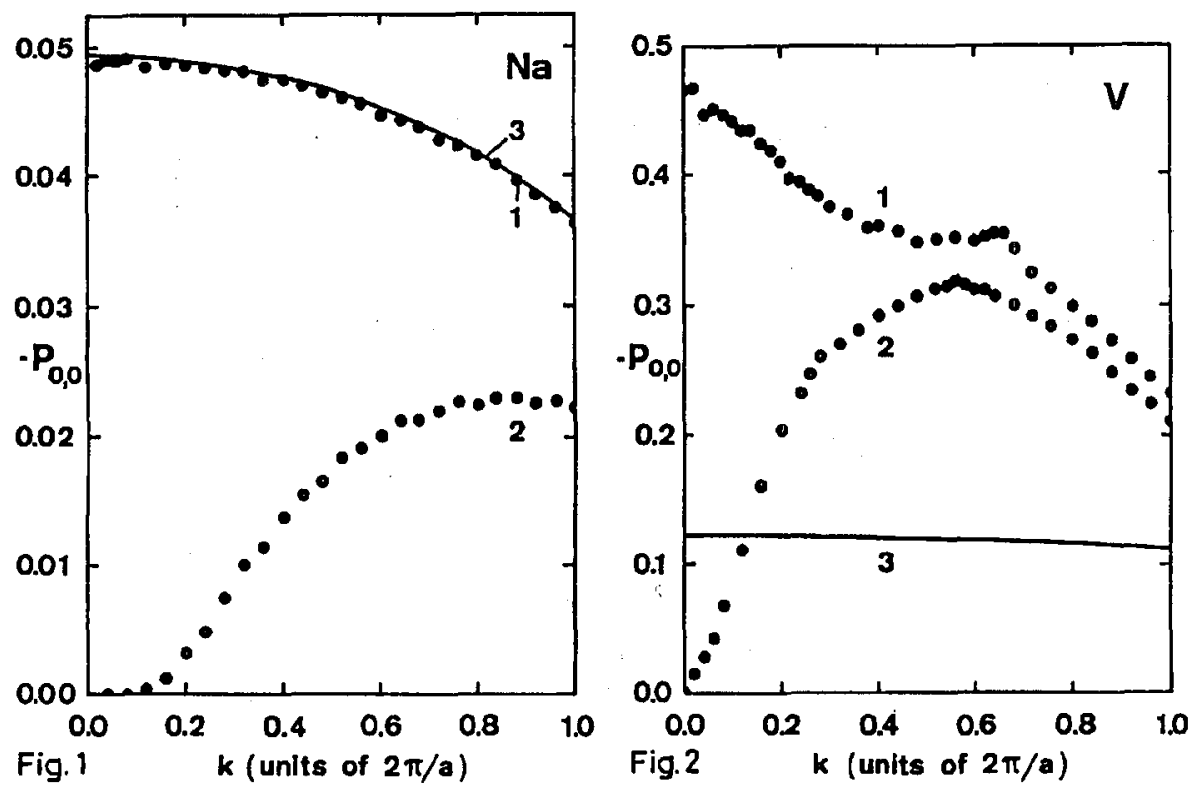

Fig. 1. Matrix element $P_{0,0}$ for $\mathrm{Na}$ as a function of $k$ along the [100] direction ( $P$ in units of (bohr $\left.{ }^{3} \mathrm{Ry}\right)^{-1}, a=8.003 \mathrm{bohr}$ ): (1) total matrix element, (2) interband contribution, (3) polarization function of the homogeneous electron gas.

Fig. 2. Matrix element $P_{0,0}$ for $\mathrm{V}$ as a function of $k$ along the [100] direction ( $P$ in units of (bohr $\left.{ }^{3} \mathrm{Ry}\right)^{-1}, a=5.54$ bohr): (1) total matrix element, (2) interband contribution, (3) polarization function of the homogeneous electron gas (5 electrons per cell).

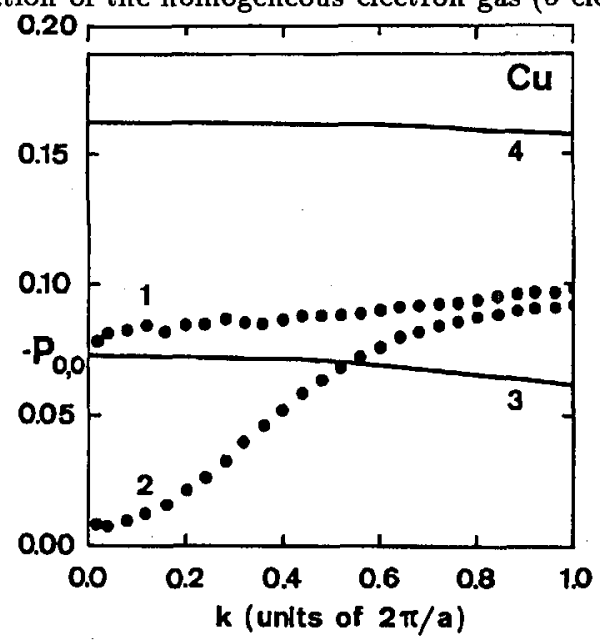

Fig. 3. Matrix element $P_{0,0}$ for $\mathrm{Cu}$ as a function of $k$ along the [100] direction ( $P$ in units of (bohr $\left.{ }^{3} \mathrm{Ry}\right)^{-1}, a=6.83 \mathrm{bohr}$ ): (1) total matrix element, (2) interband contribution, (3) polarization function of the homogeneous electron gas (1 electron per cell), (4) polarization function of the homogeneous electron gas (11 electrons per cell). 

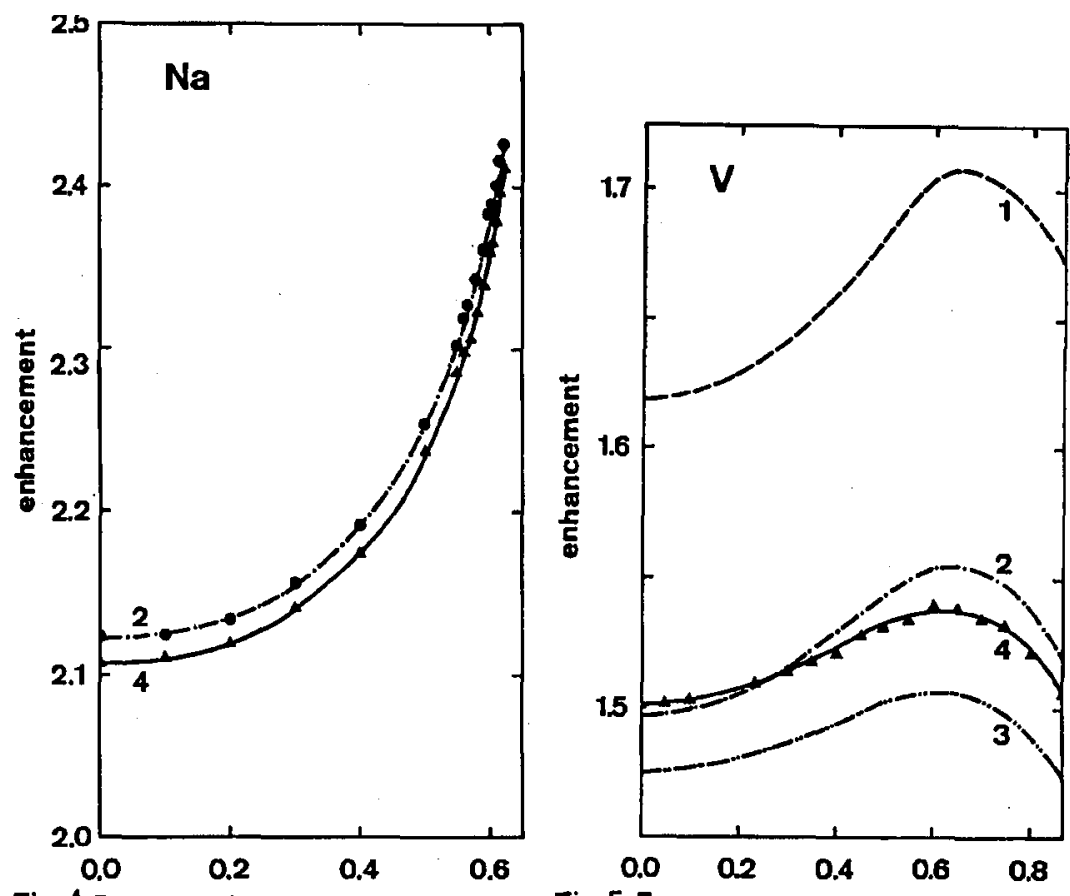

Fig. 4 「 p (units of $2 \pi / a$ ) H Fig. 5 Г

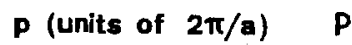

Fig. 4. Enhancement curves for $\mathrm{Na}$ as a function of $\boldsymbol{p}$ along [100]: (2) homogeneous curve, (4) curve including the full potential matrix.

Fig. 5. Enhancement curves for $\mathrm{V}$ as a function of $p$ along [111]: (1) QF-BML, (2) homogeneous curve, (3) curve including only the diagonal elements of the potential matrix, (4) curve including the full potential matrix.

found in [9] ... "the momentum dependence (for $V$ ) of both types of enhancement curves surprisingly similar". In the case of iron, we obtained the result that ... "the momentum dependence (is) somewhat more marked for the APW than for the PW excited $\mathrm{e}^{-}$and $\mathrm{e}^{+}$states" (Fig. 3 in [9]). These results are also confirmed by our new calculations on copper: as it can be seen in Figs. 6 and 7, the momentum characteristics of the QF-BML curves (1) and the corresponding homogeneous curves (2) are partly completely different, especially for the higher electron band (Figs. 6b and $7 \mathrm{~b}$ ). Along the [100] direction, the QF-BML enhancement curve of this $\mathrm{Cu}$ band shows the typical "Kahana shape". However, if Bloch scattering states are included, the $d$ characteristics of this band nearby the center of the BZ leads to a significant change of the shape of this enhancement curve.

\subsection{Bloch characteristics within the interaction potential}

In this section, we discuss results which have been achieved by numerical evaluations of the Eqs. (5), (6) and (11), that means we calculated - for the first time - BML results which include Bloch characteristics both for the electron and 


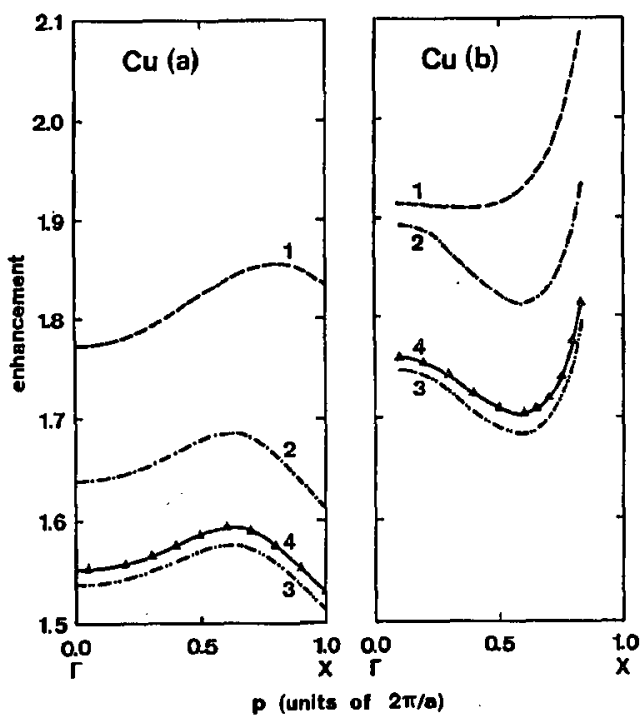

Fig. 6. Enhancement curves for $\mathrm{Cu}$ as a function of $\boldsymbol{p}$ along [100]: (a) first, (b) sixth valence band. (1) QF-BML, (2) homogeneous curve, (3) curve including only the diagonal elements of the potential matrix, (4) curve including the full potential matrix.

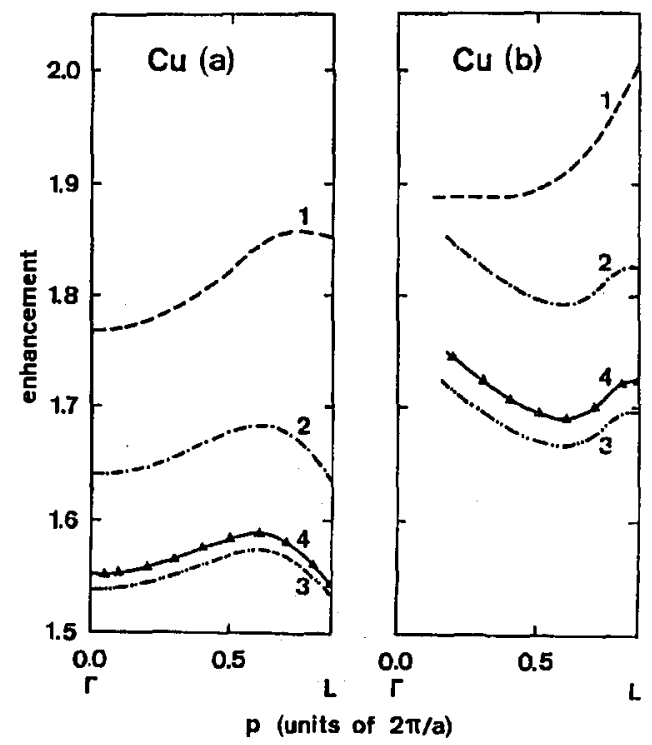

Fig. 7. Enhancement curves for $\mathrm{Cu}$ as a function of $p$ along [111]: (a) first, (b) sixth valence band. (1) QF-BML, (2) homogeneous curve, (3) curve including only the diagonal elements of the potential matrix, (4) curve including the full potential matrix. 
positron scattering states and for the electron-positron interaction potential.

Sodium (Fig. 4): The influence of the Bloch characteristics of the interaction potential is rather small due to the "nearly-free behavior" of the valence electrons of this metal. Nevertheless, the "homogeneous" enhancement curve (2) lies about one percent above the enhancement curve (4) with the full Bloch interaction included. It is interesting to mention that this effect can also be observed if one compares the enhancement curves of $\mathrm{Na}$ obtained by the QF-BML and by the LD approach, respectively (see [4], Fig. 6).

Copper (Figs. 6 and 7): One observes that the homogeneous enhancement curves (2) lie considerably above the corresponding "DE" curves (3). If the NDE are also taken into account (4), this difference reduces a little bit. The momentum dependence of the enhancement curves (2)-(4) is almost exactly the same!

Vanadium (Fig. 5): The difference between the homogeneous curve (2) and the "DE" curve (3) is smaller than in the case of $\mathrm{Cu}$. The influence of the NDE of the potential matrix is, however, much greater than for copper: the difference between the curves (2) and (3) is nearly compensated due to the NDE of the potential matrix. As a very important result one observes that the momentum dependence of the homogeneous and the inhomogeneous curve is significantly different. The inclusion of Bloch effects into the interaction potential leads to a weaker dependence of the enhancement curve on the annihilation momentum.

\section{Conclusions}

The inclusion of the potential matrix from Eq. (8) into the BML formalism influences the enhancement curves in a twofold way:

(1) The amount of the enhancement is reduced in comparison to the "homogeneous" enhancement curves. The strength of this reduction is determined by both the diagonal and the non-diagonal elements of the $v^{\text {ep }}$ matrix.

(2) The momentum dependence of the enhancement is also changed: this effect is predominantly caused by the non-diagonal elements of the potential matrix which describe the local component of the polarization of the inhomogeneous electron gas. This result corresponds with the experience that the momentum dependence of the $d$ band enhancement is often smaller for LDA results than for QF-BML results.

\section{References}

[1] J.P. Carbotte, Phys. Rev. 144, 309 (1966).

[2] H. Sormann, W. Puff, in: Positron Annihilation, Eds. P.C. Jain, R.M. Singru, K.P. Gopinathan, World Scientific, Singapore 1985, p. 161.

[3] H. Sormann, Phys. Status Solidi B 142, K45 (1987).

[4] H. Sormann, Acta Univ. Wratislav. Mat. Fiz. Astron. 56, 231 (1989).

[5] H. Sormann, in: Positron Annihilation, Ed. L. Dorikens-Vanpraet, M. Dorikens, D. Segers, World Scientific, Singapore 1989, p. 272.

[6] S. Daniuk, G. Kontrym-Sznajd, J. Mayers, A. Rubaszek, H. Stachowiak, P.A. Walters, R.N. West, J. Phys. F, Met. Phys. 17, 1365 (1987). 
[7] E. Boronski, R.N. Nieminen, Phys. Rev. B 34, 3820 (1986).

[8] T. Jarlborg, A.K. Singh, Phys. Rev. B 36, 4660 (1987).

[9] H. Sormann, D. Wallner, in: Positron Annihilation, Ed. Z. Kajcsos, Cs. Szeles, Materials Science Forum, Vol. 105-110, Trans. Tech. Publ., Switzerland 1992, p. 845.

[10] P. Genoud, Ph.D. Thesis 2442, University of Geneva, Geneva 1990.

[11] M. Taut, in: Elektronentheorie der Metalle, Ed. P. Ziesche, G. Lehmann, Springer-Verlag, Berlin 1983, p. 241ff.

[12] G. Gilat, L.J. Raubenheimer, Phys. Rev. 144, 390 (1966). 